\title{
Functional Genomics Analysis Reveals a MYC Signature Associated with a Poor Clinical Prognosis in Liposarcomas
}

Dat Tran, ${ }^{*}$ Kundan Verma, ${ }^{*}$ Kristin Ward, ${ }^{*}$ Dolores Diaz, ${ }^{*}$ Esha Kataria, ${ }^{*}$ Alireza Torabi, ${ }^{\dagger}$ Anna Almeida, ${ }^{\dagger}$ Bernard Malfoy, ${ }^{\ddagger}$ Eva W. Stratford, ${ }^{\S}$ Dianne C. Mitchell,* and Brad A. Bryan*

From the Departments of Biomedical Sciences* and Pathology, ${ }^{\dagger}$ Paul L. Foster School of Medicine, Texas Tech University Health Sciences Center, El Paso, Texas; the Curie Institute Research Center, ${ }^{\ddagger}$ Paris, France; and the Cancer Stem Cell Innovation Centre and the Department of Tumor Biology, ${ }^{\S}$ Institute of Cancer Research, Oslo University Hospital, Norwegian Radium Hospital, Oslo, Norway

\author{
Accepted for publication \\ November 20, 2014. \\ Address correspondence to \\ Brad A. Bryan, Ph.D., Paul L. \\ Foster School of Medicine, \\ Center of Excellence in Cancer \\ Research, Texas Tech Univer- \\ sity Health Sciences Center, \\ 5001 El Paso Dr., MSB1 Room \\ 2111, El Paso, TX 79905. \\ E-mail: brad.bryan@ttuhsc.edu.
}

\begin{abstract}
Liposarcomas, which are malignant fatty tumors, are the second most common soft-tissue sarcomas. Several histologically defined liposarcoma subtypes exist, yet little is known about the molecular pathology that drives the diversity in these tumors. We used functional genomics to classify a panel of diverse liposarcoma cell lines based on hierarchical clustering of their gene expression profiles, indicating that liposarcoma gene expression profiles and histologic classification are not directly correlated. Boolean probability approaches based on cancer-associated properties identified differential expression in multiple genes, including MYC, as potentially affecting liposarcoma signaling networks and cancer outcome. We confirmed our method with a large panel of lipomatous tumors, revealing that MYC protein expression is correlated with patient survival. These data encourage increased reliance on genomic features in conjunction with histologic features for liposarcoma clinical characterization and lay the groundwork for using Boolean-based probabilities to identify prognostic biomarkers for clinical outcome in tumor patients. (Am J Pathol 2015, 185: 717-728; http://dx.doi.org/10.1016/j.ajpath.2014.11.024)
\end{abstract}

Liposarcoma is the most common soft-tissue sarcoma, accounting for approximately $20 \%$ of all soft-tissue sarcoma cases. ${ }^{1}$ This tumor type is believed to arise from fat cells in deep soft tissue most commonly in patients $>40$ years and is often diagnosed only when the tumor is very large or painful. Liposarcomas fall into four major histologic subtypes (well differentiated, dedifferentiated, myxoid/round cell, and pleomorphic) and can also be classified by specific genetic alterations, including amplification of the 12q13-15 chromosomal region (well differentiated and dedifferentiated), translocation of chromosome 12 (myxoid/round cell), and a complex karyotype (pleomorphic liposarcoma). ${ }^{2-5}$ Classification of liposarcomas according to these categories tends to yield the strongest correlation to metastasis, tumor recurrence, and patient survival. For instance, well-differentiated liposarcomas exhibit low recurrence and high patient survival rates, whereas a significantly worse prognosis is attributed to more aggressive subtypes. ${ }^{6}$ Up to $25 \%$ of liposarcomas are reportedly misclassified based on histologic characterization, ${ }^{7}$ thus complicating accurate liposarcoma diagnosis and treatment response.

Owing to the high level of diagnostic discordance in classifying liposarcomas, Singer et $\mathrm{al}^{2}$ developed a systematic approach based on gene expression analysis to identify subtype-specific differentially expressed genes. Singer et $\mathrm{al}^{2}$ discovered a 142-gene predictor of tissue class based on a correlation with liposarcoma subtype. McClain et $\mathrm{al}^{8}$ used a proteomics-based approach to predict dedifferentiation in liposarcoma, reporting that protein levels of mitochondrial aldehyde dehydrogenase 2 and selenium-binding protein 1 were good predictors of dedifferentiation. Correlation of genomic profiles to metastatic risk of liposarcomas has

Supported by a Texas Tech University Health Sciences Center faculty start-up award (B.A.B.).

D.T., K.V., and K.W. contributed equally to this work.

Disclosures: None declared. 
been linked to the expression of genes involved in adipogenesis, DNA replication, mitosis, cell-cycle checkpoints, and hypoxic gene signatures. ${ }^{4,9}$ Each of these studies strictly performed supervised analyses whereby gene expression profiles were linked to the established histologic classification of liposarcomas. In contrast, Segal et $\mathrm{al}^{10}$ used unsupervised clustering to clearly demonstrate that established clinical classification of various soft-tissue sarcomas, including liposarcomas, did not exclusively reflect the hierarchical clustering patterns of their gene expression profiles. Indeed, liposarcomas were often more closely related to other diverse sarcomas than to other histologically similar liposarcomas. These data suggest that histologic and clinical characterization of liposarcomas (and other sarcomas for that matter) does not sufficiently describe the genotype and likely phenotype of the tumor, and this discrepancy may well be related to the high variability in recurrence, metastasis, treatment resistance, and survival rates observed in patients with liposarcomas.

Herein, we examine the genomic profiles of 19 cell lines reflecting the various liposarcoma histologic subtypes and used unsupervised hierarchical clustering analysis to measure relatedness between the lines based on statistical relatedness of gene expression profiles rather than histologic subtypes. We then used Boolean-based probability, functional genomics, and system-level approaches to identify statistically ranked gene networks indicative of the phenotype of each liposarcoma group. Finally, we confirmed the findings using immunohistochemical (IHC) analysis on an independent large panel of benign and malignant lipomatous tumors and in a meta-analysis against existing publically available lipomatous tumor data sets.

\section{Materials and Methods}

\section{Genomic Data Sets and Bioinformatics Analysis}

Gene expression profiles for the 19 liposarcoma cell lines were downloaded from ArrayExpress (http://www.ebi.ac.uk/ arrayexpress, Accession number E-GEOD-12972, last accessed November 17, 2014). ${ }^{11}$ These data sets were obtained using the Affymetrix GeneChip platform (Affymetrix, Santa Clara, CA) on primary human liposarcoma cell lines, including seven well-differentiated, four dedifferentiated, four pleomorphic, and four myxoid/round liposarcomas that were harvested introperatively from tumors ranging from grades I to III.

Gene expression profiles for the 105 soft-tissue sarcoma tumors used in this study were downloaded from ArrayExpress (http://www.ebi.ac.uk/arrayexpress, Accession number E-GEOD-6481, last accessed November 17, 2014). ${ }^{12}$ These data sets were obtained using the Affymetrix GeneChip platform on solid sarcoma tumors, including three lipomas, three well-differentiated liposarcomas, 15 dedifferentiated liposarcomas, 19 myxoid liposarcomas, four fibrosarcomas, six leiomyosarcomas, 21 malignant fibrous histiocytomas, three malignant peripheral nerve sheath tumors, 15 myxofibrosarcomas, and 16 synovial sarcomas.

Gene expression profiles for the 60 diverse sarcoma tumors used in this study were downloaded from ArrayExpress (http://www.ebi.ac.uk/arrayexpress, Accession number E-MEXP-2687, last accessed November 17, 2014). ${ }^{13}$ These data sets represented grade II or III angiosarcoma, leiomyosarcoma, osteosarcoma, malignant fibrous histiocytoma, malignant peripheral nerve sheath tumor, chondrosarcoma, mesenchymoma, liposarcoma, and other unclassifiable sarcomas. These tumors consisted of either primary sarcomas or tumors secondary to photon or electron beam radiation as diagnosed using the Cahan criteria. ${ }^{14}$ Pathologic diagnosis of all the tumors was previously performed using World Health Organization guidelines. All the tumor samples were obtained before chemotherapy or radiotherapy of the tumors.

The data were filtered and normalized using either GeneSpring GX software version 12.0 (Agilent Technologies, Santa Clara, CA) or Gene Cluster software version 3.0, and unsupervised hierarchical clustering with an uncentered correlation similarity metric of both the genes and arrays was performed using a centroid linkage with either GeneSpring GX or Java TreeView version 3.0. Pathway analysis to identify statistically significant gene networks and biological processes affected by the gene expression changes was performed using MetaCore software version 5.0 (Thomson Reuters, New York, NY). Protein-protein interaction networks were determined using the String database version 9.05 (http://string-db.org, last accessed November 17, 2014). Coexpression analysis was performed using GeneSpring GX.

Confirmatory meta-analysis of $M Y C$ mRNA expression was performed using the liposarcoma genomic data set previously generated by Nakayama et $\mathrm{al}^{12}$ and downloaded from ArrayExpress (http://www.ebi.ac.uk/arrayexpress, Accession number E-GEOD-6481, last accessed November 17, 2014).

\section{Boolean-Based Systems Biology Approach}

To identify statistically significant cancer-associated genes, we used a Boolean-based systems biology approach that has been previously used in applications for other cancers. ${ }^{15,16}$ In this statistical approach, we sought to rank genes based on eight functional attributes, including statistically significant gene expression alteration between the liposarcoma groups, their association as a cancer gene, if the gene product is expressed in adipogenic tissue at a detectable level, if the gene product functions as a transcription factor or excretory-secretory protein or is posttranslationally modified, and if the gene undergoes a known DNA methylation as an epigenetic modification. The rationale for the selection of each of these categories has been previously reported elsewhere. ${ }^{15}$ Using these functional attributes, we 
generated a probabilistic Boolean truth table assigning a probability value to each existing combination of Boolean variables, and this probability was derived from the proportion of cancer-associated genes exhibiting each combination of functional attributes. The cancer-associated gene list (composed of 740 genes) was defined by the Cancer Gene Census at the Sanger Centre (COSMIC, http://cancer. sanger.ac.uk/cancergenome/projects/census, last accessed November 17, 2014), the Atlas of Genetics and Cytogenetics in Oncology, ${ }^{17}$ and the disease association data of the Human Protein Reference Database. ${ }^{18}$ This trained model was then used as a guilt-by-association algorithm applied to genes statistically identified in the liposarcoma bioinformatics analysis. As described previously, ${ }^{15,19}$ the guilt-byassociation algorithm identified novel candidate genes by i) decomposing the nine Boolean functional attributes observed for the liposarcoma data set into its roots, ii) capturing the probability of each root association from the probabilistic Boolean truth table prepared for the 740 cancer-associated genes, and iii) adding the probabilities to rank the importance of the liposarcoma genes.

\section{IHC Analysis, in Situ Hybridization, and Clinical Correlation with MYC Expression}

For detection of MYC protein expression, paraffinized human lipomatous tumor tissue arrays (catalog No. IMH322; Imgenex, part of Novus Biologicals, LLC, Littleton, $\mathrm{CO}$ ) were labeled with an anti-c-MYC antibody (catalog No. ab32072; Abcam Inc., Cambridge, MA) and were quantified using alkaline phosphatase detection (Cell Marque, a Sigma-Aldrich Co., Rocklin, CA). Positive and negative controls were obtained from breast carcinoma and liposarcoma tissues, respectively. Immunopositivity was scored semiquantitatively for the percentage of tumor cells stained and staining intensity $(0$, negative; + , weak; ++ , moderate; +++ , strong). All scoring of immunostains was performed by individuals blinded to the clinical outcome for the patients. All included clinical data for the tumors, including age and sex of the patient, location of the tumor, diagnosis, and degree of differentiation, were provided by Imgenex on purchase of the lipomatous tissue arrays from the company. Histologic characterization of all the tumor sections was additionally confirmed via hematoxylin and eosin staining by the in-house Texas Tech University Health Sciences Center pathology laboratory. Scoring of MYC expression based on the IHC analysis data was correlated with clinicopathologicl parameters provided on datasheets supplied by Imgenex and with histologic diagnosis provided by Imgenex and independently confirmed by Texas Tech University Health Sciences Center pathologists. In situ hybridization to detect $M Y C$ gene amplification was performed using the MYC DNP probe (catalog No. 7601218; Ventana Medical Systems Inc., Tucson, AZ) with the BenchMark ULTRA platform (Ventana Medical
Systems Inc.) on formalin-fixed, paraffin-embedded lipomatous tumor tissue arrays (catalog No. IMH-322; Imgenex). Breast carcinoma tissue slides were used as positive controls. The ISH iView Blue Plus detection kit (catalog No. 760-097; Ventana Medical Systems Inc.) was used to detect the DNP-labeled probes.

\section{Statistical Evaluation of Patient Survival}

GraphPad Prism software version 6 (GraphPad Software Inc., San Diego, CA) was used for all the statistical calculations. Patient survival curves were generated using the Kaplan-Meier method based on the correlation between MYC expression levels (derived in the IHC studies) and survival data (provided on datasheets supplied by Imgenex). The Mantel-Cox $t$-test was used to determine significance in patient survival based on MYC expression levels, and the log-rank hazard ratio was used as a descriptive measure comparing the relative risk of death in patients depending on their MYC expression status.

\section{Results}

Unsupervised Hierarchical Clustering Analysis of Liposarcoma Gene Expression Data Sets

Daigeler et al ${ }^{11}$ previously established 19 liposarcoma cell lines from human solid tumors, including a diverse selection of well-differentiated, dedifferentiated, pleomorphic, and myxoid/round liposarcomas ranging from grades I to III (Figure 1A). These authors primarily focused on elucidating the differential gene expression between these liposarcoma cell lines after sham or doxorubicin treatment; however, we performed a meta-analysis of this publically deposited data (ArrayExpress, http://www.ebi.ac.uk/ arrayexpress, Accession number E-GEOD-12972, last accessed November 17, 2014) by excluding liposarcoma samples treated with doxorubicin and focusing on analysis of the data from the untreated tumor cell lines only. There are certain advantages to genomics analysis of liposarcoma cell lines over solid tumors that directed us to use gene expression patterns from cell lines as a starting point for this analysis. Namely, we focused solely on the expression patterns of the lipomatous tumor cells without added complications that inevitably would be introduced by the highly variable levels of infiltrating tumor stroma that exist in all solid tumors. Unsupervised hierarchical clustering analysis of the gene expression profiles of these tumor lines revealed two major groups of liposarcomas (which we assigned as group 1 or group 2) that, to our surprise, were clearly not defined based on histologic tumor subtype or grade (Figure 1B).

The use of gene expression data taken from tumor cell lines only introduces an artificiality due to engineered culture conditions, elimination of tumor microenvironment, etc. Thus, we confirmed the global gene expression data 
A

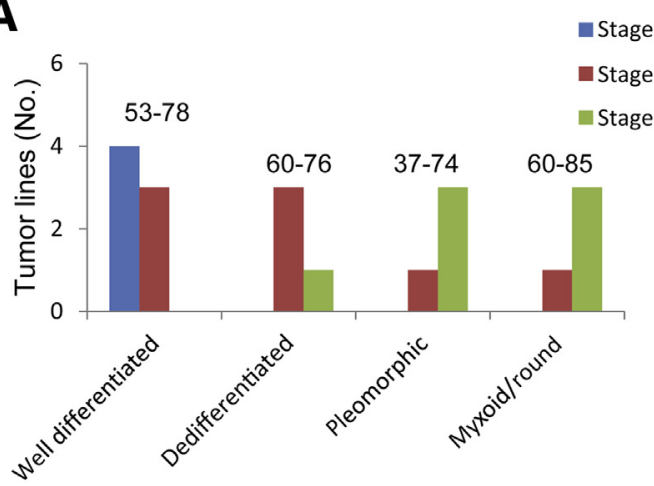

B

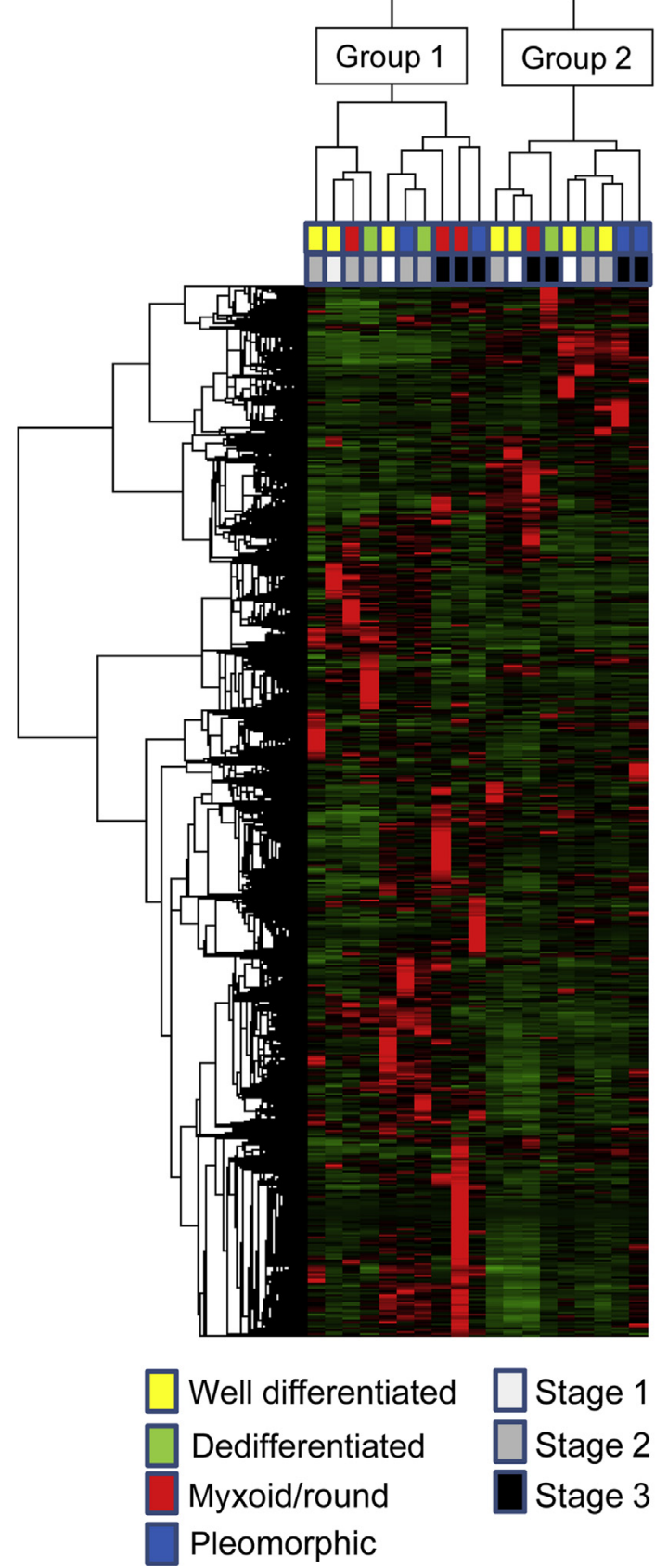

C

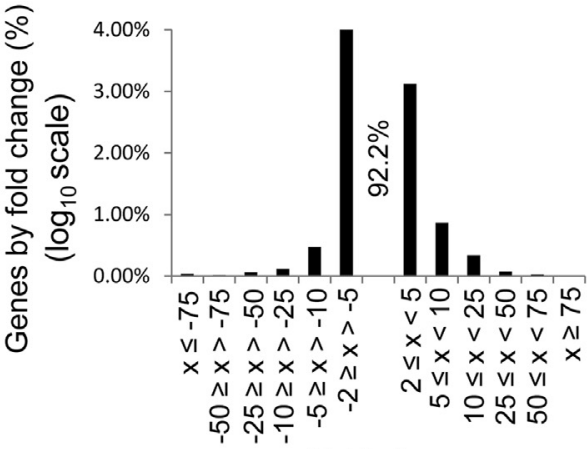

Fold change

D
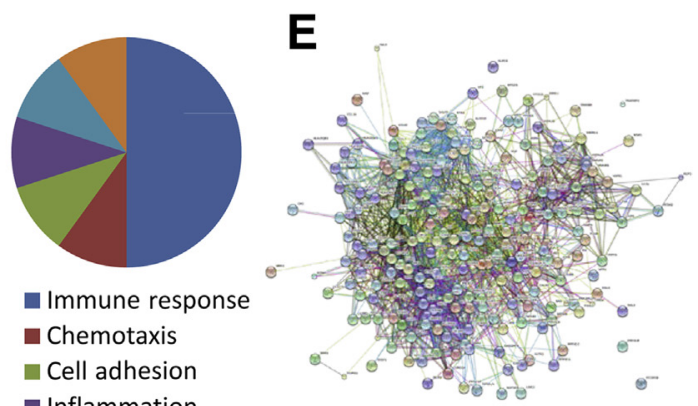

Immune response

Chemotaxis

- Cell adhesion

- Inflammation

Protein folding

- Proliferation

Figure 1 Bioinformatics classification of liposarcoma cell transcriptome patterns. A: Histologic classification and staging of the 19 liposarcoma cell lines used in the bioinformatics meta-analysis. The age ranges (in years) of the patients from which the tumors were taken are indicated above each bar. B: Unsupervised hierarchical clustering analysis of filtered and normalized liposarcoma cell gene expression data using an uncentered correlation similarity metric and centroid linkage. Histologic classification and staging are indicated for each sample according to the color chart. C: Histogram representing the amount of differential gene expression between group 1 and group 2 liposarcomas meeting the statistical criteria of twofold or greater differential expression $(P \leq 0.05)$. Of the 22,215 genes examined in this analysis, $92.2 \%$ were deemed nonsignificant. D: The list of 2047 statistically significant genes identified in this study was input into MetaCore network analysis software to identify six overrepresented process networks differing between the liposarcoma groups representing 215 select genes. E: The 215 genes were input into the String database to illustrate direct and indirect protein interactions through interconnected interaction networks. 
collected from lipomatous tumor cell lines by performing a meta-analysis of an independent genomic data set formerly publically deposited in ArrayExpress (http://www.ebi.ac.uk/ arrayexpress, Accession number E-GEOD-6481, last accessed November 17, 2014). This data set contained the global gene expression profiles of 105 soft-tissue sarcoma tumor samples, including 40 benign and malignant lipomatous tumors (although it was, unfortunately, relatively underrepresented in lipomas and well-differentiated liposarcomas). Unsupervised hierarchical clustering of these data revealed that similar to the data presented in Figure 1 based solely on liposarcoma tumor cell lines, a diverse array of sarcoma tumors revealed divergent gene expression clustering patterns that did not always reflect the histologic classification of the tumor sample (Supplemental Figure S1). In the lipomatous tumors there existed two clusters of lipomas (both relatively closely clustered); three clusters of well-differentiated liposarcomas (which were closely related to lipomas, dedifferentiated liposarcomas, and a single malignant fibrous histiocytoma); six clusters of dedifferentiated lipomatous tumors (although the two largest clusters were closely related and may equate to only five clusters depending on the interpretation of the data); and three very unrelated clusters of myxoid liposarcoma tumors. The results of the present meta-analysis of both lipomatous tumor cell lines and sarcoma tumor samples agree with data presented by Segal et al, ${ }^{10}$ where whole gene expression analysis on a panel of 51 diverse pathologically confirmed adult soft-tissue sarcomas revealed that histologic classification of particular sarcoma tumor types was not by any means reflective of their unique hierarchical clustering patterns of global gene expression. Furthermore, we see similar divergent hierarchical clustering patterns on unsupervised clustering of genomic data collected from 60 sarcoma tumor microarray profiles deposited in ArrayExpress (http://www.ebi.ac.uk/arrayexpress, Accession number E-MEXP-2687, last accessed November 17, 2014) ${ }^{13}$ (Supplemental Figure S2), suggesting that conflicts between histologic and genomic classification extend beyond lipomatous tumors to a diverse number of sarcomas in general. In this meta-analysis, we revealed that sarcomas can be clearly divided into two groups: one that contains angiosarcomas, leiomyosarcomas, and osteosarcomas and another that contains primarily sarcomas that are pleiomorphic in nature and often difficult to accurately classify based on histology alone. The single lipomatous tumor included in this analysis (a well-differentiated liposarcoma) clustered with the more pleomorphic tumors.

\section{Identification of Key Genetic Processes Differing between the Liposarcoma Groups}

Of the 22,215 genes whose expression was analyzed in each of the 19 lipomatous tumor cell lines, differential expression (a twofold or greater change, $P \leq 0.05$ ) was observed for 2047 genes, with 979 up-regulated and 1068 down-regulated based
Table 1 Top 10 Process Networks that Differentiate Group 1 and Group 2 Liposarcomas as Determined by MetaCore Analysis

\begin{tabular}{ll}
\hline Process network & $P$ value \\
\hline Immune response-phagocytosis & $1.90 \times 10^{-11}$ \\
Immune response-phagosome & $3.44 \times 10^{-9}$ \\
$\quad$ in antigen presentation & \\
Immune response-BRC pathway & $5.57 \times 10^{-8}$ \\
Chemotaxis & $1.70 \times 10^{-7}$ \\
Cell adhesion-leukocytes & $3.88 \times 10^{-7}$ \\
Inflammation-histamine signaling & $4.93 \times 10^{-7}$ \\
Immune response-Th17-derived cytokines & $6.15 \times 10^{-7}$ \\
Immune response-antigen presentation & $6.40 \times 10^{-7}$ \\
Protein folding-folding under normal conditions & $8.31 \times 10^{-7}$ \\
Proliferation-positive regulation cell & $8.39 \times 10^{-7}$ \\
$\quad$ proliferation & \\
\hline
\end{tabular}

on average intensity values of group 2 relative to group 1 (Figure 1C and Supplemental Table S1). We sought to understand the genetic networks that differentiate the two liposarcoma groups identified in this analysis. Owing to the overwhelmingly large number of gene expression changes observed between the two liposarcoma groups, we narrowed the candidate list by inputting the 2047 differentially expressed genes into MetaCore network analysis software, identifying the top 10 most statistically relevant gene process networks most differentially regulated between the groups. Identification of these process networks narrowed the number of candidates to 215 highly relevant genes that were primarily involved in immune response, inflammation, chemotaxis, cell adhesion, protein folding, and proliferation (Table 1, Supplemental Tables S2-S7, and Figure 1D). Input of these 215 genes into the String database, which identifies direct and indirect protein interactions based on data obtained from genomic context, high-throughput experiments, coexpression, and data mining, revealed a remarkably interconnected network of interactions (Figure 1E).

\section{Using a Boolean Implications Network Approach to Statistically Rank the Importance of Key Genes}

Fold change ranking of gene expression data is commonly used by researchers to identify the importance of differentially expressed genes between one or more defined groups of samples. However, a small gene expression change in a major oncogene or tumor suppressor could have a significantly more dramatic effect on the tumor phenotype than, for instance, a large gene expression change for a less important gene. Without in-depth empirical knowledge of each of the 215 genes identified in the analysis, it is difficult to determine in an unbiased manner which gene expression changes represent the most important alterations associated with each liposarcoma group. We, therefore, implemented a Boolean logic scheme derived from cancer-associated genes and developed a guilt-by-association algorithm that was 


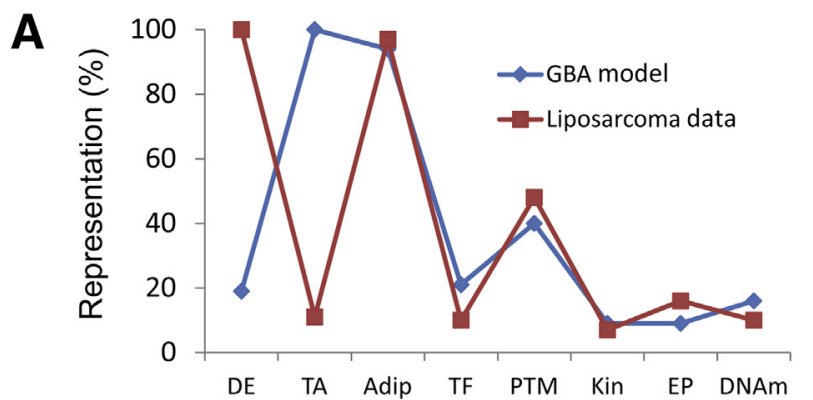

B
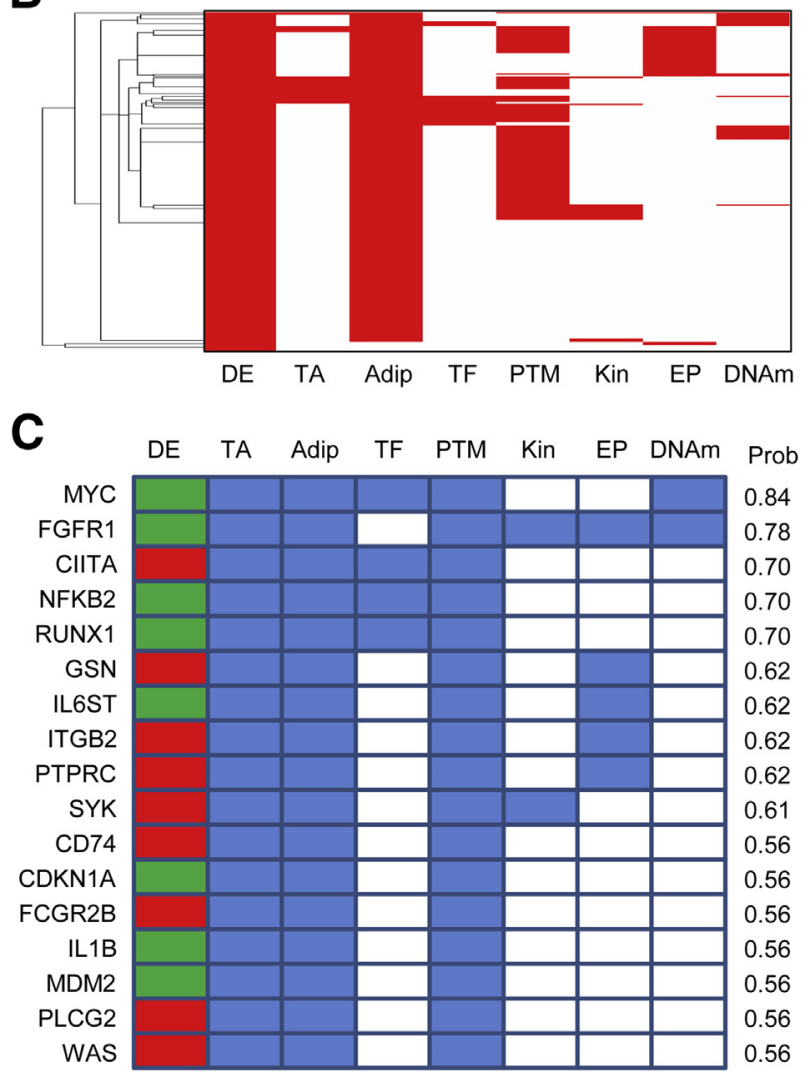

Figure 2 Boolean-based approach to predict cancer-associated genes in the liposarcoma data set. A: Line chart comparing the distribution of binarized Boolean variables for the 740 genes in the guilt-by-association (GBA) model versus the 215 genes selected from the liposarcoma data set. B: Unsupervised clustering of the 215 genes based on their distribution of the binarized variables used in the Boolean probability analysis. C: Each of the 215 genes was ranked based on the eight functional attributes used in the Boolean probability analysis. Shown are the 17 genes whose Boolean scores were $\geq 0.50$. Red and green for differentially expressed (DE) correlate to overexpression and underexpression, respectively, of the gene in group 2 relative to group 1 liposarcomas. Blue for all the other variables correlates to a 1 , and white equals a 0 regarding binarized Boolean attributes. Adip, known expression in adipocyte tissue; DNAm, DNA methylation; EP, excreted protein; Kin, kinase; Prob, Boolean probability score; PTM, posttranslational modification; TA, tumor-associated gene; TF, transcription factor.

subsequently applied to the list of 215 genes identified in this analysis. This strategy has been previously used to identify with statistical confidence cancer-associated genes across multiple cancer types. ${ }^{15,16,19}$ To begin, we developed a training set from 740 known cancer-associated genes and assigned binarized Boolean variables based on known differential expression in cancer, tumor association, adipocytelineage tissue expression, posttranslational modifications, DNA methylation of the gene, or whether the gene product is a kinase or is secreted into the extracellular matrix to generate a probabilistic truth table (Supplemental Table S8). This truth table represented the probabilities from the proportion of cancer-associated genes presenting a particular profile of $0 \mathrm{~s}$ and $1 \mathrm{~s}$ across the variables. We then used extensive literature and database mining to assign binarized Boolean variables to each of the 215 genes identified in the analysis of the liposarcoma data sets. A comparison of the percentage of representation for the binarized variables of the 740 genes in the guilt-by-association model compared with the 215 genes identified in the present analysis is shown in Figure 2A. An association heat map of the distribution of the binarized variables across the 215 genes identified in the liposarcoma data set is shown in Figure 2B. For each of the 215 genes, the particular combination of the Boolean variables was decomposed into its roots, the probability associated with each root was captured from the probabilistic Boolean truth table, and the probabilities were added to rank the importance of the 215 genes (Figure 2C). Nagaraj and Reverter ${ }^{15}$ demonstrated in their analysis that cancer genes received an average Boolean score of 0.22 compared with an average score of 0.08 for noncancerassociated genes, thus demonstrating the validity of this approach. To identify only the most statistically significant genes (and efficiently reduce the working numbers of genes being studied) we eliminated any of the 214 genes with a $<0.5$ Boolean probability score, leaving 17 genes with scores ranging from 0.56 to 0.84 (Figure 2C). Of these genes, eight were down-regulated (MYC, FGFR1, NFKB2, RUNX1, IL6ST, CDKN1A, ILIB, and MDM2) and nine were up-regulated (CIITA, GSN, ITGB2, PTPRC, SYK, CD74, $F C G R 2 B, P L C G 2$, and $W A S$ ) in group 2 liposarcomas relative to group 1 tumors. Of all the genes ranked via Boolean analysis, MYC exhibited the highest score (0.84).

\section{A MYC Signature Is Associated with a Subset of Malignant Liposarcomas}

MYC has been referred to as the "oncogene from hell" by Soucek and $\operatorname{Evan}^{20}$ owing to its role in promoting cell-cycle progression, self-renewal of cells, tumor expansion, and genomic instability through playing a dual role as a transcription factor for $>15 \%$ of the genome and a chromatin remodeler. ${ }^{21}$ Point mutations, translocations, and amplifications in the $M Y C$ gene have all been reported across various cancers, and enhanced MYC activity is strongly associated with poor prognosis. ${ }^{22}$ Based on liposarcoma group averages, MYC mRNA expression was up-regulated in the present analysis by an average of 3.5 -fold in group 1 versus group 2 liposarcoma tumors. Examination of $M Y C$ mRNA expression on a tumor line by tumor line basis revealed that the expression of this gene is relatively high in 

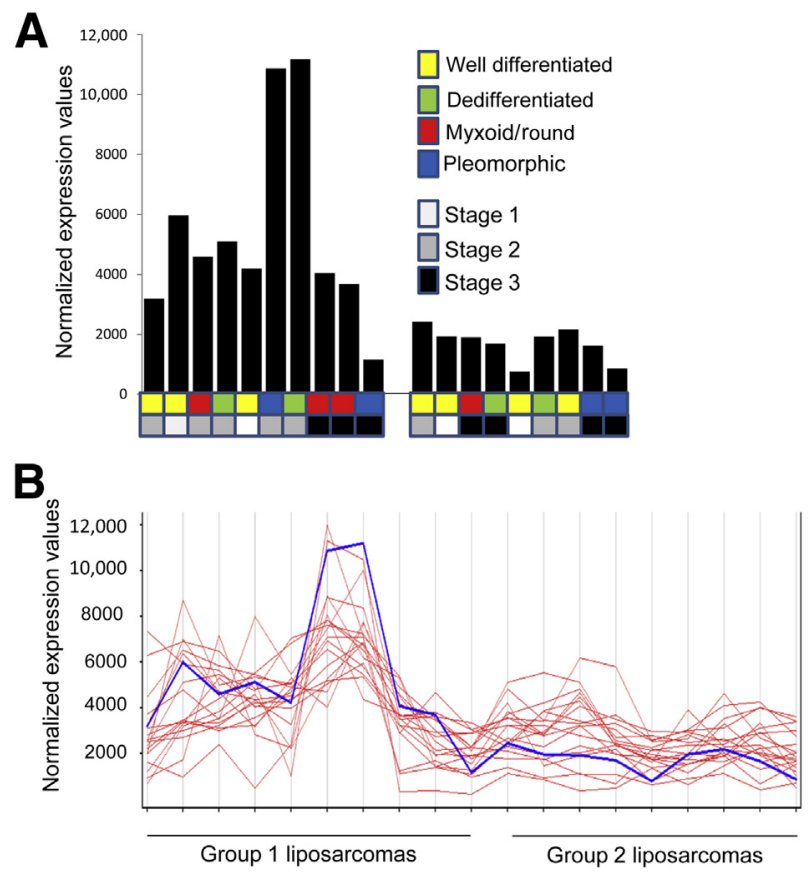

Figure 3 Examination of $M Y C$ and coexpressed gene expression across liposarcoma cell transcriptional profiles. A: Normalized gene expression values for MYC mRNA across individual group 1 and group 2 liposarcoma tumor cells based on meta-analysis of the liposarcoma gene expression data set. Histologic classification and staging are indicated for each sample according to the color chart. B: Coexpression analysis of the 2047 differentially expressed genes reveals 19 genes whose normalized expression values statistically significantly correlated to that of MYC mRNA across all 19 liposarcoma cell lines. Blue line, MYC expression; red lines, expression values of the 19 statistically coexpressed genes.

9 of 10 group 1 tumors and relatively low in $100 \%$ of group 2 tumors (Figure 3A). Coexpression analysis uncovered 19 genes whose expression statistically significantly correlated with $M Y C$ expression, including MARCKSL1, CAPRIN1, BTG1, RBM14, RBM4, CCT6A, DHX15, EIF4E, DKC1, SFPQ, TRIB1, DHX9, TDO2, NXT2, METAP2, FKBP1A, DHX9, STIPI, and SRRT (Figure 3B).

We performed IHC staining for MYC protein expression on 52 lipomatous tumors, including benign lipomas to the full spectrum of malignant well-differentiated, dedifferentiated, pleiomorphic, and myxoid liposarcomas (Table 2 and Figure 4, A and B). Weak to no expression of this protein was observed in benign lipoma tumors. MYC protein expression was observed at various levels in all the malignant liposarcoma tumors tested. As a general rule, the level of MYC expression across the tumor panel was largely independent of liposarcoma histologic subtype, although well-differentiated liposarcomas exhibited a slightly higher ratio of MYC low tumors than did more aggressive liposarcoma tumor subtypes. We further sought to independently corroborate the data by performing a metaanalysis examining $M Y C$ mRNA expression in a publically available liposarcoma genomic data set ${ }^{12}$ downloaded from ArrayExpress (http://www.ebi.ac.uk/arrayexpress, Accession No. E-GEOD-6481, last accessed November 17,
2014). Examination of the normalized microarray intensity values in these data revealed that MYC expression was low for lipomas and well-differentiated liposarcomas and extremely variable for more malignant tumors ranging from very low to very high expression (Figure $4 \mathrm{C}$ ). To further confirm the validity of the Boolean probability analysis, we also performed IHC analysis on RUNX1 (which demonstrated a Boolean probability score of 0.7 ), revealing that immunoreactivity and staining intensity were closely correlated between MYC and RUNX1 (Supplemental Table S9), with a Pearson correlation coefficient of 0.764 . This indicates a very strong correlation between the expression of each protein.

The chromosome 8 region where the $M Y C$ gene is located has been identified as one of the most commonly amplified regions of the genome. ${ }^{23}$ Various amplifications, deletions, and gene fusions have been extensively reported across various subtypes of liposarcomas, ${ }^{24}$ but no reports have previously indicated that the $M Y C$ oncogene is amplified in this tumor type. Given the relatively higher expression of MYC in a subset of liposarcomas, we used in situ hybridization to evaluate the possibility that amplification of the $M Y C$ gene is contributing to these observations. Although the positive control (breast carcinoma) revealed strong $M Y C$ reactivity in the tumor cells, not a single incident of $M Y C$ amplification was detected across 52 lipoma and liposarcoma tumor sections tested (Figure 4, D and E). These data indicate that the differential expression of the $M Y C$ gene in this analysis is not due to gene amplification.

\section{MYC Expression in Liposarcomas Correlates with Patient Survival}

Deregulation of MYC expression is a key driver for many aggressive cancers. Because this analysis points to MYC expression as being highly variable across liposarcomas, we sought to correlate MYC expression with clinical characteristics such as patient mortality and survival rates. Using deidentified clinical data from lipomatous tumor patients whose tumors were used for the IHC analysis detailed in Table 2 we correlated MYC protein levels with patient survival rates by generating Kaplan-Meier survival curves. Lipomatous tumors exhibiting no MYC protein staining correlated with $100 \%$ patient survival, whereas steadily increasing MYC protein expression inversely correlated with patient survival $(+, 84 \%$ survival;,$++ 59 \%$ survival; ,$+++ 25 \%$ survival) (Figure 5). For statistical analysis of the survival data set, we combined MYC negative and lowexpressing patients together as the experimental control group given that only 2 of 52 tumors tested exhibited no MYC expression. Comparison of patient survival in the high and moderate MYC-expressing tumors, with that in the no/ low MYC-expressing tumors demonstrated significant differences based on the Mantel-Cox $t$-test $\left(P_{\text {moderate }}=0.0294\right.$; $\left.P_{\text {high }}<0.0001\right)$. Log-rank hazard ratios measuring the relative risk of patient mortality were calculated to be 3.6 for 
Table 2 MYC Expression in Human Lipomatous Tumors

\begin{tabular}{|c|c|c|c|c|c|c|}
\hline Age, years & Sex & Organ & Diagnosis & Degree of differentiation & Tissue type & MYC score \\
\hline 37 & $\mathrm{~F}$ & Neck, soft tissue & Lipoma & NA & Benign & + \\
\hline 56 & M & Back, soft tissue & Lipoma & NA & Benign & + \\
\hline 42 & $\mathrm{~F}$ & Shoulder, soft tissue & Lipoma & NA & Benign & 0 \\
\hline 53 & $\mathrm{~F}$ & Neck, soft tissue & Lipoma & NA & Benign & 0 \\
\hline 60 & M & Breast & Lipoma & NA & Benign & + \\
\hline 51 & $\mathrm{~F}$ & Thigh, soft tissue & Lipoma & NA & Benign & + \\
\hline 55 & $\mathrm{~F}$ & Breast & Lipoma & NA & Benign & + \\
\hline 42 & $\mathrm{~F}$ & Scalp, soft tissue & Lipoma & NA & Benign & + \\
\hline 40 & $\mathrm{~F}$ & Thigh, soft tissue & Liposarcoma & Well differentiated & Malignant & + \\
\hline 49 & $\mathrm{~F}$ & Thigh, soft tissue & Liposarcoma & Well differentiated & Malignant & + \\
\hline 56 & M & Neck, soft tissue & Liposarcoma & Well differentiated & Malignant & + \\
\hline 33 & M & Calf, soft tissue & Liposarcoma & Well differentiated & Malignant & + \\
\hline 67 & M & Retroperitoneum, soft tissue & Liposarcoma & Well differentiated & Malignant & + \\
\hline 50 & $\mathrm{~F}$ & Axilla, soft tissue & Liposarcoma & Well differentiated & Malignant & + \\
\hline 28 & M & Retroperitoneum, soft tissue & Liposarcoma & Well differentiated & Malignant & + \\
\hline 70 & $\mathrm{~F}$ & Shoulder, soft tissue & Liposarcoma & Well differentiated & Malignant & ++ \\
\hline 48 & $\mathrm{~F}$ & Buttock, soft tissue & Liposarcoma & Well differentiated & Malignant & + \\
\hline 56 & $\mathrm{~F}$ & Mesentery & Liposarcoma & Well differentiated & Malignant & ++ \\
\hline 44 & $\mathrm{~F}$ & Mediastinum, soft tissue & Liposarcoma & Well differentiated & Malignant & ++ \\
\hline 42 & $\mathrm{~F}$ & Thigh, soft tissue & Liposarcoma & Well differentiated & Malignant & + \\
\hline 73 & $\mathrm{~F}$ & Axilla, soft tissue & Liposarcoma & Well differentiated & Malignant & + \\
\hline 33 & $\mathrm{~F}$ & Pelvic cavity, soft tissue & Liposarcoma & Myxoid & Malignant & ++ \\
\hline 44 & M & Retroperitoneum, soft tissue & Liposarcoma & Myxoid & Malignant & +++ \\
\hline 47 & M & Axilla, soft tissue & Liposarcoma & Myxoid & Malignant & +++ \\
\hline 78 & M & Thigh, soft tissue & Liposarcoma & Myxoid & Malignant & + \\
\hline 64 & M & Abdominal cavity, soft tissue & Liposarcoma & Myxoid & Malignant & ++ \\
\hline 45 & M & Abdomen, soft tissue & Liposarcoma & Myxoid & Malignant & ++ \\
\hline 74 & $\mathrm{~F}$ & Thigh, soft tissue & Liposarcoma & Myxoid & Malignant & ++ \\
\hline 43 & M & Thigh, soft tissue & Liposarcoma & Myxoid & Malignant & ++ \\
\hline 41 & M & Peritoneal cavity, soft tissue & Liposarcoma & Myxoid & Malignant & + \\
\hline 59 & M & Chest, soft tissue & Liposarcoma & Pleomorphic & Malignant & +++ \\
\hline 66 & $\mathrm{~F}$ & Abdominal cavity, soft tissue & Liposarcoma & Pleomorphic & Malignant & +++ \\
\hline 70 & $\mathrm{~F}$ & Abdominal cavity, soft tissue & Liposarcoma & Pleomorphic & Malignant & + \\
\hline 53 & M & Retroperitoneum, soft tissue & Liposarcoma & Dedifferentiated & Malignant & +++ \\
\hline 61 & $\mathrm{~F}$ & Abdominal cavity, soft tissue & Liposarcoma & Dedifferentiated & Malignant & ++ \\
\hline 55 & M & Abdominal cavity, soft tissue & Liposarcoma & Dedifferentiated & Malignant & + \\
\hline 61 & M & Abdominal cavity, soft tissue & Liposarcoma & Dedifferentiated & Malignant & +++ \\
\hline
\end{tabular}

F, female; M, male; NA, not applicable; 0 , negative; + , weak; ++ , moderate; +++ , strong.

MYC moderate-expressing tumors and 9.0 for MYC highexpressing tumors relative to the no/low MYC-expressing tumors, indicating that mortality risk in patients with lipomatous tumors is statistically significantly correlated with increasing MYC expression levels. Median survival time was calculated for patients with high MYC-expressing tumors 27.5 months after diagnosis (median survival time was undefined in MYC moderate-expressing lipomatous tumors 


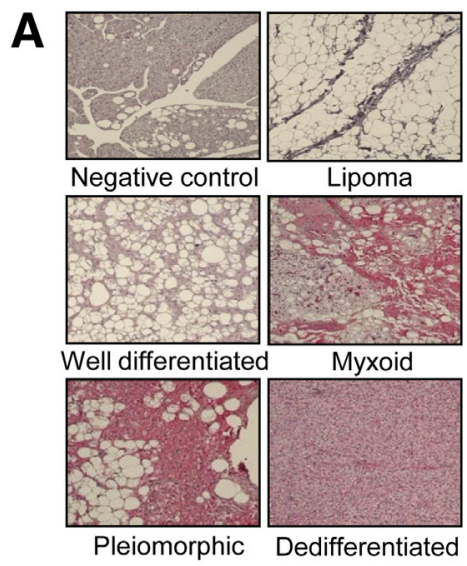

C

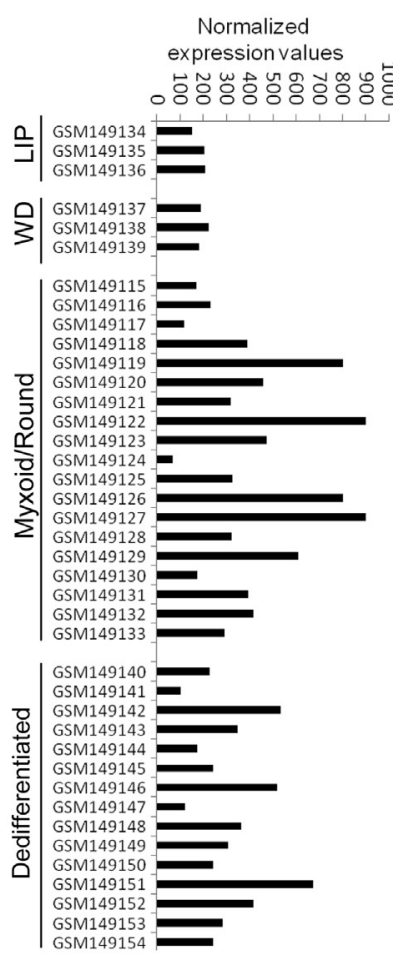

B

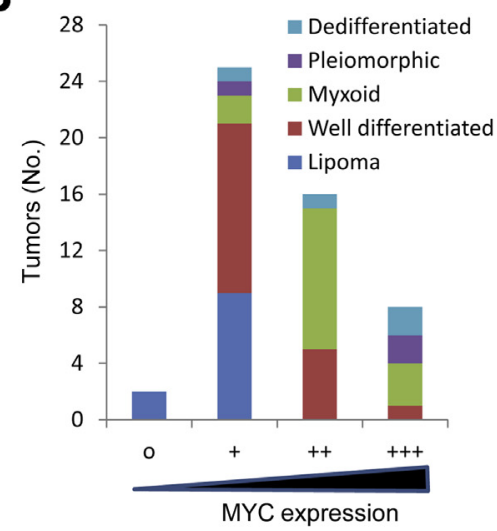

D

\section{E}

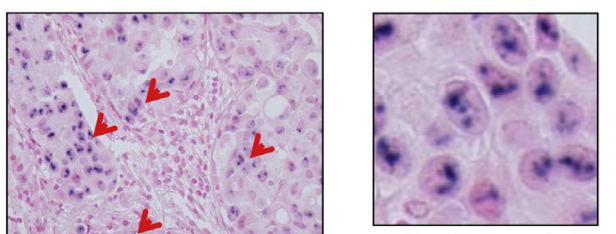

Positive control

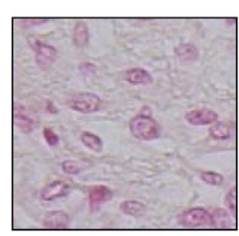

Liposarcoma
Figure 4 Variable MYC expression is observed in independent analysis of liposarcoma tumor samples. A: Immunohistochemical analysis was used to detect MYC protein levels in 52 lipomatous tumor sections representing lipomas and each of the histologic subtypes of liposarcomas. Representative images from the negative control and stained sections of lipoma and liposarcoma histologic subtypes are shown. B: Histogram illustrating the distribution of MYC protein levels across 52 lipomatous tumors based on tissue staining and intensity. C: Independent analysis of the normalized $M Y C$ gene expression via a meta-analysis of a previously published liposarcoma genomic data set (Gene Expression Omnibus; $h$ ttps:// www.ncbi.nlm.nih.gov/geo; Accession number GSE6481) representing lipomas and three liposarcoma subtypes. D: Representative images of in situ hybridization to detect MYC gene amplification across 52 lipoma and liposarcoma tumors. Breast cancer tumors were used as a positive control. Red arrows indicate MYC gene amplification. E: Highpower magnification images of MYC in situ hybridization. Original magnification: $\times 1000$ (E). LIP, lipoma; WD, well-differentiated liposarcomas. because more than half of the patients survived). Collectively, these data suggest that the level of MYC protein expression is strongly correlated with clinical outcome in liposarcoma patients.

\section{Discussion}

Liposarcomas are morphologically classified into four main subtypes: well differentiated, dedifferentiated, myxoid/ round cell, and pleomorphic. ${ }^{25}$ Despite the historically established clinical classification system for liposarcomas based on histologic features, the data presented herein clearly suggest that the gene expression profiles of liposarcomas as a whole do not correlate based on histologic type or clinical staging. Considering that chemotherapeutic and radiation sensitivity varies considerably between each liposarcoma subtype,${ }^{26}$ it is imperative for effective treatment of patients to accurately classify liposarcomas.

A review of $>160$ lipoma and liposarcoma tumors that were previously classified solely on histologic characterization resulted in $23 \%$ of the tumors being reclassified based on cytogenetic information. ${ }^{7}$ Several studies have used supervised clustering to link gene and protein expression signatures to established liposarcoma histologic classification $^{2,4,8,9}$; however, given the high percentage of liposarcoma patient misdiagnosis reported in the literature, it is questionable whether these approaches would yield data that accurately reflect the phenotype and genotype of the individual tumors. Segal et $\mathrm{al}^{10}$ used unsupervised hierarchical clustering of gene expression data to demonstrate that liposarcomas did not clearly cluster according to histologic characterization. Consistently, heterogeneity in gene expression patterns has also been shown for other soft-tissue 


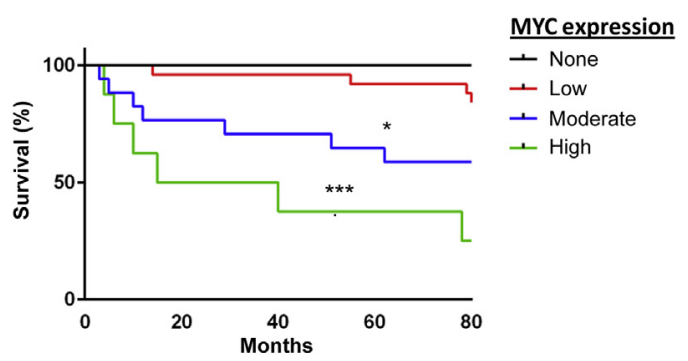

Figure 5 Correlation of MYC expression with clinical outcome in liposarcoma patients. Kaplan-Meier plot correlating MYC protein expression levels to lipomatous tumor patient survival $(N=52$ lipomatous tumors). The Mantel-Cox $t$-test was used to compare no/low MYCexpressing tumors with moderate or high MYC-expressing tumors. ${ }^{*} P<0.05,{ }^{* * *} P<0.001$.

sarcomas, independent of knowledge of the clinical features of the tumor, ${ }^{27}$ and sarcomas that previously were believed to be different tumor types have been shown to be indistinguishable based on genomic profiles and shared prognostic markers. ${ }^{28}$

This leads to the question of why would histologically similar liposarcomas display such dissimilar gene expression profiles. The most common objection that has been presented by scientists and clinicians to defend the sanctity of histologic classification of liposarcomas is that gene expression profiles are variable based on features such as anatomical location or the influence of the stromal microenvironment. Thus, when performing gene expression profiling on solid tumors, these uncontrollable variables will adversely affect and muddy the interpretation of the data and might explain hierarchical clustering patterns that do not closely correlate with histologic classification. The present study sought to overcome these issues by performing hierarchical clustering on gene expression profiles obtained from liposarcoma cell lines grown in culture-independent of their anatomical location or stromal influences. These data revealed hierarchical clustering of gene expression patterns that did not closely align with the current clinical classification system. We further confirmed the cell line data using meta-analysis of genomic profiles of solid tumors collected from a diverse group of sarcomas, including 40 lipomatous tumors. Collectively, the data from the report and others point to the need for a radical reevaluation of liposarcoma classification (and sarcomas in general) based on a combination of histologic and genomic features. Indeed, the World Health Organization has made a step in this direction by modifying their classification system to include undifferentiated high-grade pleomorphic sarcoma for sarcoma tumors without evidence of differentiation. This has led to a reclassification of malignant fibrous histiocytoma, which is the most common form of sarcoma in humans, although the present data suggest that more aggressive modification of this classification system is necessary to accurately reflect the diverse nature of many sarcoma tumors, including liposarcomas.
Rieker et $\mathrm{al}^{29}$ performed unsupervised hierarchical clustering of chromosomal imbalances identified using comparative genomic hybridization in liposarcomas, revealing two major liposarcoma clusters each composed of various subgroups. Similarly, the present analysis using gene expression profiling revealed two major liposarcoma clusters with statistically significant expression differences in 2047 genes. Because this was an overwhelming number of genes to study, we used bioinformatics network analysis to select the most statistically relevant genes, identifying 215 key genes involved in immune response, inflammation, chemotaxis, cell adhesion, protein folding, and proliferation. At this point, the standard accepted approach would be to use empirical knowledge and/or to rely on fold change to identify the key genes that distinguished these two liposarcoma groups; however, such an approach can lead to experimental bias based on the highest fold changes and/or the limits of an individual's knowledge. We sought to use a less biased approach to identify the most important genes in the data set by developing a Boolean-based systems approach that could be applied to liposarcoma gene expression data sets. This statistical approach to identify the most relevant genes was based on estimating weights for the functional attributes associated with known cancer genes, combining those weights using a Boolean logic scheme, and calculating the probability-based rank associates with each of the 215 differentially regulated genes identified in the network analysis. This reduced the number of gene candidates to a manageable 17 genes, each with strong Boolean probability scores correlating their functional attributes to those of a training set of established cancer-associated genes. Indeed, all 17 genes have been shown to be major regulators of the oncogenic state across diverse tumor types, strongly suggesting that between these two groups of liposarcomas there is a fundamental genetic difference in tumor signaling that could be diagnostically and/or therapeutically exploited.

MYC plays an integral role in a variety of cellular processes, including proliferation, metabolism, cell adhesion, differentiation, and apoptosis, and it is estimated to regulate the expression of approximately $15 \%$ of all genes through functioning as a classical transcription factor via binding to enhancer box sequences and regulating global chromatin structure via recruiting histone acetyltransferases. ${ }^{21,30}$ The MYC gene resides on human chromosome 8 , and amplification of this region is widespread across many cancers and is a negative prognostic factor for patient outcome. ${ }^{31}$ Despite the statistically higher expression of $M Y C$ in group 1 liposarcomas, the present in situ hybridization experiments did not detect $M Y C$ amplification in any of the lipomatous tumors that were tested, indicating that differential $M Y C$ expression between the groups could be due to other factors, such as deregulated transcriptional regulation or alterations in mRNA stability. Correlation of MYC protein expression with clinical data on human patients diagnosed as having lipomatous tumors revealed strong 
statistical correlations linking high MYC expression with reduced overall patient survival rates. Although positive reactivity for MYC protein expression in lipomatous tumors statistically significantly correlated with decreased patient survival and increased time to mortality, it is by no means absolute because even tumors with strong staining $(+++)$ for MYC resulted in patient mortality only $75 \%$ of the time. This finding suggests that solely relying on MYC expression may be limited in its applicability, and further studies that develop multigene algorithms using the genes identified in the present Boolean-based approach or examination of the 19 MYC co-expressed genes identified in this study could be used to develop a superior prognostic biomarker panel for liposarcoma patient outcome with increased accuracy.

In summary, this study used bioinformatics-based metaanalysis to provide genomic evidence to challenge the accuracy of the current histology-based liposarcoma classification system. We used a novel unbiased statistical approach to identify a subset of key tumor-associated genes that differentiate two major groups of liposarcomas. This approach proved remarkably fruitful in that it identified the $M Y C$ oncogene as a potential prognostic biomarker whose expression can be statistically significantly correlated to clinical outcome in liposarcoma patients.

\section{Acknowledgments}

D.T. and K.V. performed the meta-analysis of liposarcoma data and Boolean statistics; D.D. performed the MYC IHC staining; K.W. analyzed the MYC IHC staining; E.K. performed statistical analysis of the IHC correlations; A.T. provided consultation of histology; A.A. generated the sarcoma genomic data sets; B.M. generated the sarcoma genomic data sets and wrote and revised the manuscript; E.W.S. designed the experiments and wrote and revised the manuscript; D.C.M. performed the bioinformatics analysis, designed the experiments, and wrote and revised the manuscript; B.A.B. designed the experiments, performed the statistical analysis of the clinical data, and wrote and revised the manuscript.

\section{Supplemental Data}

Supplemental material for this article can be found at $h t t p: / /$ dx.doi.org/10.1016/j.ajpath.2014.11.024.

\section{References}

1. Mack TM: Sarcomas and other malignancies of soft tissue, retroperitoneum, peritoneum, pleura, heart, mediastinum, and spleen. Cancer 1995, 75:211-244

2. Singer S, Socci ND, Ambrosini G, Sambol E, Decarolis P, Wu Y, O’Connor R, Maki R, Viale A, Sander C, Schwartz GK, Antonescu CR: Gene expression profiling of liposarcoma identifies distinct biological types/subtypes and potential therapeutic targets in well-differentiated and dedifferentiated liposarcoma. Cancer Res 2007 67:6626-6636

3. Willmore-Payne C, Holden J, Turner KC, Proia A, Layfield LJ: Translocations and amplifications of chromosome 12 in liposarcoma demonstrated by the LSI CHOP breakapart rearrangement probe. Arch Pathol Lab Med 2008, 132:952-957

4. Francis P, Namlos HM, Muller C, Eden P, Fernebro J, Berner JM, Bjerkehagen B, Akerman M, Bendahl PO, Isinger A, Rydholm A, Myklebost O, Nilbert M: Diagnostic and prognostic gene expression signatures in 177 soft tissue sarcomas: hypoxia-induced transcription profile signifies metastatic potential. BMC Genomics 2007, 8:73

5. Gebhard S, Coindre JM, Michels JJ, Terrier P, Bertrand G, Trassard M, Taylor S, Chateau MC, Marques B, Picot V, Guillou L: Pleomorphic liposarcoma: clinicopathologic, immunohistochemical, and follow-up analysis of 63 cases: a study from the French Federation of Cancer Centers Sarcoma Group. Am J Surg Pathol 2002, 26:601-616

6. Singer S, Antonescu CR, Riedel E, Brennan MF: Histologic subtype and margin of resection predict pattern of recurrence and survival for retroperitoneal liposarcoma. Ann Surg 2003, 238:358-370; discussion $370-371$

7. de Vreeze RS, de Jong D, Nederlof PM, Ariaens A, Tielen IH, Frenken L, Haas RL, van Coevorden F: Added value of molecular biological analysis in diagnosis and clinical management of liposarcoma: a 30-year single-institution experience. Ann Surg Oncol 2010, 17:686-693

8. McClain CM, Friedman DB, Hajri T, Coffin CM, Cates JM: Predicting dedifferentiation in liposarcoma: a proteomic approach. Virchows Arch 2013, 463:85-92

9. Gobble RM, Qin LX, Brill ER, Angeles CV, Ugras S, O’Connor RB, Moraco NH, Decarolis PL, Antonescu C, Singer S: Expression profiling of liposarcoma yields a multigene predictor of patient outcome and identifies genes that contribute to liposarcomagenesis. Cancer Res 2011, 71:2697-2705

10. Segal NH, Pavlidis P, Antonescu CR, Maki RG, Noble WS, DeSantis D, Woodruff JM, Lewis JJ, Brennan MF, Houghton AN, Cordon-Cardo C: Classification and subtype prediction of adult soft tissue sarcoma by functional genomics. Am J Pathol 2003, 163: 691-700

11. Daigeler A, Klein-Hitpass L, Chromik MA, Muller O, Hauser J, Homann HH, Steinau HU, Lehnhardt M: Heterogeneous in vitro effects of doxorubicin on gene expression in primary human liposarcoma cultures. BMC Cancer 2008, 8:313

12. Nakayama R, Nemoto T, Takahashi H, Ohta T, Kawai A, Seki K, Yoshida T, Toyama Y, Ichikawa H, Hasegawa T: Gene expression analysis of soft tissue sarcomas: characterization and reclassification of malignant fibrous histiocytoma. Mod Pathol 2007, 20:749-759

13. Hadj-Hamou NS, Lae M, Almeida A, de la Grange P, Kirova Y, Sastre-Garau X, Malfoy B: A transcriptome signature of endothelial lymphatic cells coexists with the chronic oxidative stress signature in radiation-induced post-radiotherapy breast angiosarcomas. Carcinogenesis 2012, 33:1399-1405

14. Cahan WG, Woodard HQ, Higinbotham NL, Stewart FW, Coley BL: Sarcoma arising in irradiated bone: report of 11 cases. Cancer 1948, 1: $3-29$

15. Nagaraj SH, Reverter A: A Boolean-based systems biology approach to predict novel genes associated with cancer: application to colorectal cancer. BMC Syst Biol 2011, 5:35

16. Shmulevich I, Dougherty ER, Kim S, Zhang W: Probabilistic Boolean Networks: a rule-based uncertainty model for gene regulatory networks. Bioinformatics 2002, 18:261-274

17. Huret JL, Dessen P, Bernheim A: Atlas of genetics and cytogenetics in oncology and haematology, year 2003. Nucleic Acids Res 2003, 31: 272-274

18. Keshava Prasad TS, Goel R, Kandasamy K, Keerthikumar S, Kumar S, Mathivanan S, Telikicherla D, Raju R, Shafreen B, Venugopal A, Balakrishnan L, Marimuthu A, Banerjee S, Somanathan DS, Sebastian A, Rani S, Ray S, Harrys Kishore CJ, Kanth S, Ahmed M, 
Kashyap MK, Mohmood R, Ramachandra YL, Krishna V, Rahiman BA, Mohan S, Ranganathan P, Ramabadran S, Chaerkady R, Pandey A: Human Protein Reference Database-2009 update. Nucleic Acids Res 2009, 37:D767-D772

19. Mukherjee S, Pelech S, Neve RM, Kuo WL, Ziyad S, Spellman PT, Gray JW, Speed TP: Sparse combinatorial inference with an application in cancer biology. Bioinformatics 2009, 25:265-271

20. Soucek L, Evan G: Myc: is this the oncogene from Hell? Cancer Cell 2002, 1:406-408

21. Gearhart J, Pashos EE, Prasad MK: Pluripotency redux: advances in stem-cell research. N Engl J Med 2007, 357:1469-1472

22. Nesbit CE, Tersak JM, Prochownik EV: MYC oncogenes and human neoplastic disease. Oncogene 1999, 18:3004-3016

23. Courjal F, Cuny M, Simony-Lafontaine J, Louason G, Speiser P, Zeillinger R, Rodriguez C, Theillet C: Mapping of DNA amplifications at 15 chromosomal localizations in 1875 breast tumors: definition of phenotypic groups. Cancer Res 1997, 57: $4360-4367$

24. Conyers R, Young S, Thomas DM: Liposarcoma: molecular genetics and therapeutics. Sarcoma 2011, 2011:483154
25. Hameed M: Pathology and genetics of adipocytic tumors. Cytogenet Genome Res 2007, 118:138-147

26. Rhomberg W: The radiation response of sarcomas by histologic subtypes: a review with special emphasis given to results achieved with razoxane. Sarcoma 2006, 2006:87367

27. Skubitz KM, Pambuccian S, Manivel JC, Skubitz AP: Identification of heterogeneity among soft tissue sarcomas by gene expression profiles from different tumors. J Transl Med 2008, 6:23

28. Carneiro A, Francis $\mathrm{P}$, Bendahl PO, Fernebro J, Akerman M, Fletcher C, Rydholm A, Borg A, Nilbert M: Indistinguishable genomic profiles and shared prognostic markers in undifferentiated pleomorphic sarcoma and leiomyosarcoma: different sides of a single coin? Lab Invest 2009, 89:668-675

29. Rieker RJ, Weitz J, Lehner B, Egerer G, Mueller A, Kasper B, Schirmacher P, Joos S, Mechtersheimer G: Genomic profiling reveals subsets of dedifferentiated liposarcoma to follow separate molecular pathways. Virchows Arch 2010, 456:277-285

30. Chen Y, Olopade OI: MYC in breast tumor progression. Expert Rev Anticancer Ther 2008, 8:1689-1698

31. Dang CV: MYC on the path to cancer. Cell 2012, 149:22-35 